\title{
Fully automated convolutional neural network-based affine algorithm improves liver registration and lesion co-localization on hepatobiliary phase T1-weighted MR images
}

Kyle A. Hasenstab ${ }^{1,2+}$, Guilherme Moura Cunha ${ }^{1,6^{*}+}$ (D), Atsushi Higaki ${ }^{1}$, Shintaro Ichikawa ${ }^{1}$, Kang Wang ${ }^{1,2}$, Timo Delgado', Ryan L. Brunsing ${ }^{3}$, Alexandra Schlein ${ }^{1}$, Leornado Kayat Bittencourtt, Armin Schwartzman ${ }^{5}$, Katie J. Fowler ${ }^{1}$, Albert Hsiao ${ }^{2}$ and Claude B. Sirlin ${ }^{1}$

\begin{abstract}
Background: Liver alignment between series/exams is challenged by dynamic morphology or variability in patient positioning or motion. Image registration can improve image interpretation and lesion co-localization. We assessed the performance of a convolutional neural network algorithm to register cross-sectional liver imaging series and compared its performance to manual image registration.

Methods: Three hundred fourteen patients, including internal and external datasets, who underwent gadoxetate disodium-enhanced magnetic resonance imaging for clinical care from 2011 to 2018, were retrospectively selected. Automated registration was applied to all 2,663 within-patient series pairs derived from these datasets. Additionally, 100 within-patient series pairs from the internal dataset were independently manually registered by expert readers. Liver overlap, image correlation, and intra-observation distances for manual versus automated registrations were compared using paired $t$ tests. Influence of patient demographics, imaging characteristics, and liver uptake function was evaluated using univariate and multivariate mixed models.
\end{abstract}

Results: Compared to the manual, automated registration produced significantly lower intra-observation distance $(p<0.001)$ and higher liver overlap and image correlation $(p<0.001)$. Intra-exam automated registration achieved 0.88 mean liver overlap and 0.44 mean image correlation for the internal dataset and 0.91 and 0.41 , respectively, for the external dataset. For inter-exam registration, mean overlap was 0.81 and image correlation 0.41 . Older age, female sex, greater inter-series time interval, differing uptake, and greater voxel size differences independently reduced automated registration performance $(p \leq 0.020)$.

Conclusion: A fully automated algorithm accurately registered the liver within and between examinations, yielding better liver and focal observation co-localization compared to manual registration.

Keywords: Gadolinium ethoxybenzyl DTPA, Image processing (computer assisted), Liver, Magnetic resonance imaging, Neural networks (computer)

\footnotetext{
*Correspondence: gcunha@ucsd.edu

${ }^{\dagger}$ The authors Kyle A Hasenstab and Guilherme Moura Cunha contributed equally to this work.

'Liver Imaging Group, Department of Radiology, University of California San

Diego, La Jolla, CA, USA

${ }^{6}$ Altman Clinical Translational Research Institute, 9452 Medical Center Drive,

Lower Level 501, La Jolla, CA 92037, USA

Full list of author information is available at the end of the article
} 


\section{Key points}

- Image registration across series can improve lesion co-localization and reader confidence

- Combining convolutional neural network-based segmentation with affine transformations created a fully automated three-dimensional registration method for magnetic resonance images of the liver

- This algorithm improved liver overlap and focal liver observation co-localization over standard manual registration

\section{Background}

Characterization of focal liver observations (i.e., areas distinctive from background liver representing either lesions or pseudolesions) [1] requires synthesis of imaging features across multiple contrast-enhanced phases and/ or series and often demands incorporation of data across exams acquired at multiple time points. Proper spatial alignment during and between exams is challenged by the dynamic morphology of the liver and variability in patient positioning, body habitus, and physiological motion [2-4]. For instance, differences in respiratory phase may shift the liver position by as much as $30 \mathrm{~mm}$ between acquired images [5-8]. Such shifts can significantly reduce radiologists' ability to co-localize observations across series, especially when there are multiple observations and/or exams [9-11].

Most radiologists in clinical practice rely on manual, and rigid registration of images (i.e., scrolling through a stack of images to find the most similar slice position for comparison) acquired at different time points, which can be time-consuming and achieves partial alignment only in the slice direction. Alignment across the entire liver volume is not possible in routine clinical practice, and separate alignments may be required for each observation. The need for repeated alignment is tedious, slows workflow, introduces opportunities for alignment errors, and potentially contributes to interpretive mistakes. By making images acquired at different time points, positions, or modalities geometrically similar, image registration can improve observation colocalization and reader confidence $[12,13]$.

Affine and deformable liver-focused medical image registration algorithms have been proposed to address these challenges but typically require operator supervision and are slowed by intensive processing and computing requirements [14-16]. Also, most algorithms have been evaluated only in small patient cohorts without generalization and have not been adopted in clinical radiology practice $[10,11,17-19]$.

Convolutional neural networks $(\mathrm{CNN})$ have been used in deformable registration tasks [20-24] and can potentially overcome some of these barriers. However, since the nonlinear transformations of deformable registration can cause anatomical distortions, affine registration algorithms (which restrict image transformations to scaling, rotation, translation, and shearing) are generally preferred for diagnostic imaging [25, 26]. Studies have shown that liver registration is improved by first segmenting the liver to create a liver mask and then using the mask rather than the whole image as input into the registration algorithm [14, 17]. CNNs can provide the required segmentation and potentially improve the accuracy of the liver registration. We combined these technical advances to create a fast, fully automated 3D affine registration algorithm for liver imaging that incorporates CNN-based liver segmentation.

The purpose of this study was to assess the performance of the proposed fully automated 3D affine algorithm to register intra- and inter-exam liver imaging series and to compare its performance to standard manual image registration. Secondary purposes were to confirm that incorporating $\mathrm{CNN}$-based liver segmentation improves registration performance, to compare registration performance using different types of $\mathrm{CNN}$-segmented liver masks, and to show scalability and generalizability of the results. As an exploratory aim, we performed cross-modality (CT to MRI) and multiphase (arterial phase to hepatobiliary phase MRI) registration as proof of concept.

\section{Methods \\ Design}

In this retrospective dual-center study, we included gadoxetate disodium-enhanced three-dimensional (3D) T1-weighted hepatobiliary phase (HBP) magnetic resonance imaging (MRI) studies in adult patients for clinical care. A subset of images from an internal dataset was used to conduct a small-scale reader substudy, which compared the performance of the algorithm to manual registration performed by expert radiologists. The complete set of images from the internal dataset was used to show algorithm scalability and identify factors that affect registration performance (large-scale substudy). An external dataset was used to show algorithm generalizability (external validation substudy). The overall study design is shown in Fig. 1. The study was Health Insurance Portability and Accountability Act compliant and approved by the institutional review board. Informed consent was waived.

\section{Image data}

The internal image dataset comprised images from patients with chronic liver disease undergoing two or more gadoxetate-enhanced liver MRI examinations at 1.5 or 3 T (General Electric Medical Systems, WI, USA) for hepatocellular carcinoma surveillance at our institution from 2011 to 2018. These images were 3D fat-saturated T1-weighted gradient-echo series, acquired $15 \mathrm{~min}$ or later after contrast injection (HBP images). The contrast agent was intravenously administered at the dose of 


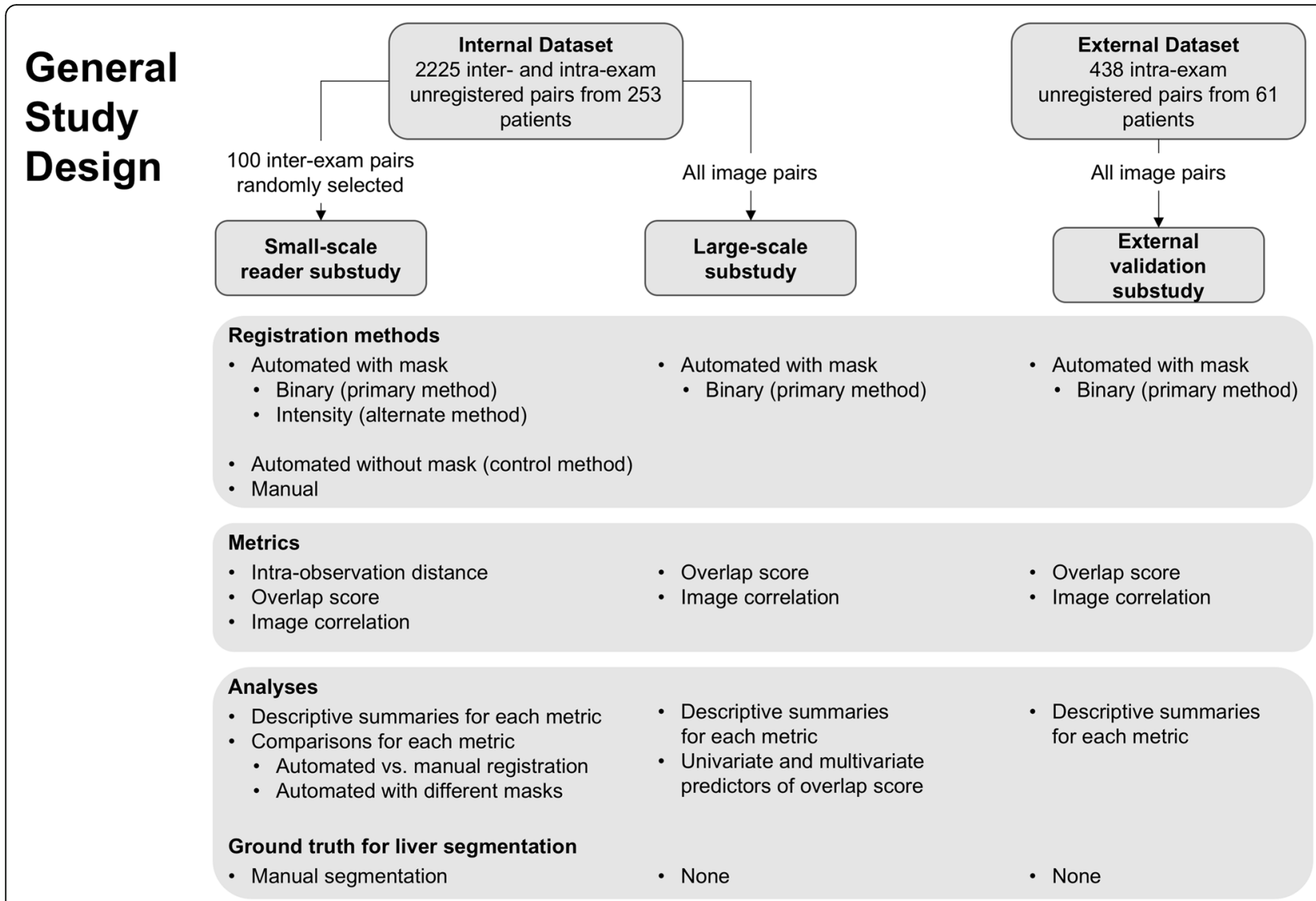

Fig. 1 Study design

$0.025 \mathrm{mmol} / \mathrm{kg}$. Baseline and follow-up studies of 253 unique patients provided a total of 564 unique HBP series because some patients had multiple HBP series acquired at different delays after contrast injection in a given examination. All inter-exam series pairs (different examinations) and intra-exam pairs (different HBP series acquired within the same examination) produced a total of 2,225 possible within-patient series pairs. Table 1 summarizes patient and imaging characteristics.

As part of another study [27], series in the internal dataset were reviewed by two abdominal fellowshiptrained radiologists (A.H. and G.M.C.), with 5 and 10 years of experience in liver imaging, who independently determined the presence of imaging artifacts and additionally classified each series as A (adequate HBP contrast uptake) or B (suboptimal HBP contrast uptake). Series were classified according to the Liver Imaging Reporting and Data System [1] as adequate if the liver was unequivocally more hyperintense than hepatic blood vessels and suboptimal if this criterion was not met. Discordant classifications were adjudicated in consensus.

The external image dataset comprised T1-weighted 3D fat-saturated gradient-echo HBP acquisitions from patients who underwent gadoxetate disodium-enhanced 1.5-T liver
MRI examinations (Siemens Healthcare, Erlangen, Germany) for various clinical indications at an outpatient imaging center outside the USA (Diagnósticos da América SA [DASA]) from September to November 2018. A predosed syringe with $10 \mathrm{ml}(0.25 \mathrm{mmol} / \mathrm{ml})$ of gadoxetate disodium-based contrast was infused by peripheral IV at a rate of $1 \mathrm{ml} / \mathrm{s}$. This dataset included intra-exam series pairs only, since full anonymization of patient information prior to data transfer precluded tracking exams acquired at different dates to the same individual. Sixty-one unique patients provided 192 unique HBP series with 438 possible within-patient series pairs. Patient characteristics for this cohort were not available.

For the exploratory aim, we selected by convenience from the internal dataset one patient (a 47-year-old male) with contemporaneous contrast-enhanced computed tomography $(\mathrm{CT}$ ) and one patient (a 78-year-old female) with arterial-phase 3D T1-weighted images acquired in the same exam as the HBP images.

\section{Fully automated affine registration algorithm}

The registration algorithm comprised a previously developed liver segmentation CNN [28] and an affine transformation network executed on a workstation with a Titan V 
Table 1 Demographic and imaging characteristics of the 253 patients in the internal dataset as well as their effects on liver overlap score

\begin{tabular}{|c|c|c|c|c|c|c|c|c|}
\hline \multirow[b]{2}{*}{ Stratum } & \multicolumn{2}{|c|}{ Demographic summary } & \multicolumn{3}{|c|}{ Univariate analysis } & \multicolumn{3}{|c|}{ Multivariate analysis } \\
\hline & $\begin{array}{l}\text { Number of image } \\
\text { pairs }\end{array}$ & $\begin{array}{l}\text { Number of } \\
\text { patients }\end{array}$ & Coefficient & $95 \% \mathrm{Cl}$ & $p$ & Coefficient & $95 \% \mathrm{Cl}$ & $p$ \\
\hline Overall & 2,225 & 253 & 0.86 & {$[0.85,0.87]$} & $<0.001$ & & & \\
\hline Age $^{*}$ (years) & $\begin{array}{l}58.38 \pm 12.37 \\
(38.60-77)\end{array}$ & & & & & & & \\
\hline$<45$ (intercept) & 260 & 33 & 0.89 & {$[0.85,0.92]$} & $<0.001$ & / & / & / \\
\hline $45-64$ & 1,211 & 142 & -0.02 & {$[-0.06,0.02]$} & 0.34 & -0.01 & {$[-0.04,0.02]$} & 0.56 \\
\hline$>65$ & 754 & 78 & -0.05 & {$[-0.09,-0.01]$} & 0.02 & -0.04 & {$[-0.07,0.00]$} & 0.06 \\
\hline \multicolumn{9}{|l|}{ Sex } \\
\hline M (intercept) & 1,062 & 108 & 0.87 & {$[0.86,0.89]$} & $<0.001$ & / & / & / \\
\hline F & 1,163 & 145 & -0.03 & {$[-0.05,0.00]$} & 0.02 & -0.03 & {$[-0.05,-0.01]$} & 0.01 \\
\hline $\mathrm{BMI}^{*}\left(\mathrm{~kg} / \mathrm{m}^{2}\right)$ & $\begin{array}{l}20.06 \pm 5.99 \\
(20.65-39.53)\end{array}$ & & & & & & & \\
\hline$<24.9$ (intercept) & 571 & 62 & 0.83 & {$[0.81,0.86]$} & $<0.001$ & & & \\
\hline $25-30$ & 792 & 91 & +0.03 & {$[-0.00,0.06]$} & 0.05 & & & \\
\hline$>30$ & 842 & 94 & +0.04 & {$[0.01,0.07]$} & 0.01 & & & \\
\hline \multicolumn{9}{|l|}{ Etiology } \\
\hline Hepatitis B (intercept) & 466 & 37 & 0.85 & {$[0.82,0.89]$} & $<0.001$ & & & \\
\hline Hepatitis C & 1,042 & 108 & +0.01 & {$[-0.02,0.05]$} & 0.50 & & & \\
\hline Alcohol & 164 & 33 & -0.01 & {$[-0.06,0.04]$} & 0.63 & & & \\
\hline $\begin{array}{l}\text { Nonalcoholic fatty liver } \\
\text { disease }\end{array}$ & 276 & 35 & +0.02 & {$[-0.02,0.07]$} & 0.39 & & & \\
\hline Autoimmune hepatitis & 98 & 11 & +0.03 & {$[-0.03,0.10]$} & 0.34 & & & \\
\hline Other & 179 & 29 & +0.00 & {$[-0.05,0.05]$} & 0.93 & & & \\
\hline $\begin{array}{l}\text { Time between series* } \\
\text { (days) }\end{array}$ & $\begin{array}{l}324.72 \pm 407.73 \\
(0-1,255)\end{array}$ & & & & & & & \\
\hline Same day (intercept) & 674 & 196 & 0.9 & {$[0.89,0.92]$} & $<0.001$ & / & / & / \\
\hline $0-1$ year & 823 & 135 & -0.05 & {$[-0.06,-0.04]$} & $<0.001$ & -0.03 & {$[-0.04,-0.02]$} & $<0.001$ \\
\hline $1-2$ years & 469 & 93 & -0.08 & {$[-0.09,-0.07]$} & $<0.001$ & -0.05 & {$[-0.07,-0.03]$} & $<0.001$ \\
\hline$>2$ years & 259 & 29 & -0.10 & {$[-0.12,-0.08]$} & $<0.001$ & -0.08 & {$[-0.10,-0.06]$} & $<0.001$ \\
\hline \multicolumn{9}{|l|}{ Artifacts } \\
\hline No artifacts (intercept) & 1,278 & 185 & 0.86 & {$[0.85,0.88]$} & $<0.001$ & & & \\
\hline One image & 597 & 105 & -0.00 & {$[-0.02,0.01]$} & 0.68 & & & \\
\hline Both images & 350 & 65 & -0.01 & {$[-0.02,0.01]$} & 0.55 & & & \\
\hline \multicolumn{9}{|l|}{$\begin{array}{l}\text { HBP contrast uptake } \\
\text { adequacy }\end{array}$} \\
\hline $\begin{array}{l}\text { Both adequate } \\
\text { (intercept) }\end{array}$ & 1,435 & 193 & 0.87 & {$[0.86,0.88]$} & $<0.001$ & / & / & / \\
\hline $\begin{array}{l}\text { One adequate, } \\
\text { one suboptimal }\end{array}$ & 350 & 54 & -0.02 & {$[-0.03,-0.01]$} & 0.01 & -0.02 & {$[-0.03,0.00]$} & 0.01 \\
\hline Both suboptimal & 340 & 62 & +0.02 & {$[-0.01,0.04]$} & 0.15 & +0.00 & {$[-0.02,0.02]$} & 0.96 \\
\hline Missing & 100 & 16 & / & / & / & / & / & / \\
\hline $\begin{array}{l}\text { Voxel volume difference } \\
\left(\mathrm{mm}^{3}\right)\end{array}$ & $\begin{array}{l}0.76 \pm 1.52 \\
(0-3.02)\end{array}$ & & & & & & & \\
\hline 0 (intercept) & 746 & 186 & 0.90 & {$[0.89,0.92]$} & $<0.001$ & / & / & / \\
\hline $0-1$ & 1,064 & 141 & -0.06 & {$[-0.07,-0.05]$} & $<0.001$ & -0.02 & {$[-0.03,-0.01]$} & $<0.001$ \\
\hline$>1$ & 402 & 86 & -0.09 & {$[-0.10,-0.07]$} & $<0.001$ & -0.05 & {$[-0.06,-0.03]$} & $<0.001$ \\
\hline
\end{tabular}


Table 1 Demographic and imaging characteristics of the 253 patients in the internal dataset as well as their effects on liver overlap score (Continued)

\begin{tabular}{|c|c|c|c|c|c|c|}
\hline \multirow[b]{2}{*}{ Stratum } & \multicolumn{2}{|c|}{ Demographic summary } & \multicolumn{2}{|l|}{ Univariate analysis } & \multicolumn{2}{|l|}{ Multivariate analysis } \\
\hline & $\begin{array}{l}\text { Number of image } \\
\text { pairs }\end{array}$ & $\begin{array}{l}\text { Number of } \\
\text { patients }\end{array}$ & Coefficient $95 \% \mathrm{Cl}$ & $p$ & Coefficient $95 \% \mathrm{Cl}$ & $p$ \\
\hline Slice thickness* $(\mathrm{mm})$ & $\begin{array}{l}4.53 \pm 0.73 \\
(3.00-6.00)\end{array}$ & & & & & \\
\hline Pixel spacing* (mm) & $\begin{array}{l}0.82 \pm 0.15 \\
(0.70-0.94)\end{array}$ & & & & & \\
\hline
\end{tabular}

$B M I$ body mass index, $H B P$ hepatobiliary phase

* Mean +/- standard deviation; 5 th and 95 th percentiles in parentheses

graphics processing unit (NVIDIA, CA, USA) and implemented on the Keras Application Program Interface (API) [29], a widely used open-source toolkit for deep neural network implementation.

For inter-exam registration, the HBP series from the baseline exam served as the "static" series while the HBP series from the follow-up exam served as "moving" series. For intra-exam registration, the earlier HBP series after contrast injection served as the "static" series whereas the later HBP series served as "moving" series. For the exploratory aim, the CT series or the arterialphase MR series served as the moving series.

Automated registration of each pair of static and moving series was accomplished in two steps (Fig. 2). First, the CNN segmented the liver on each series to create liver masks. Second, the liver masks were registered using the affine transformation network. The affine transformation parameters were then applied to the whole images, not just the liver masks, to map the moving series to the static series space. Both the liver segmentation $\mathrm{CNN}$ and affine transformation network are described further below.

\section{Convolutional neural network for liver segmentation}

The liver segmentation model is a two-dimensional $\mathrm{CNN}$ with U-Net model architecture trained to segment T1-weighted HBP images and previously validated using liver volumetry and proton density fat fraction estimations [28]. Axial slices from 3D HBP series were individually sent through the liver segmentation network to produce a set of liver masks (one per slice), which were then concatenated and post-processed to form a 3D binary liver mask. For additional details on the development and implementation of the liver segmentation $\mathrm{CNN}$,

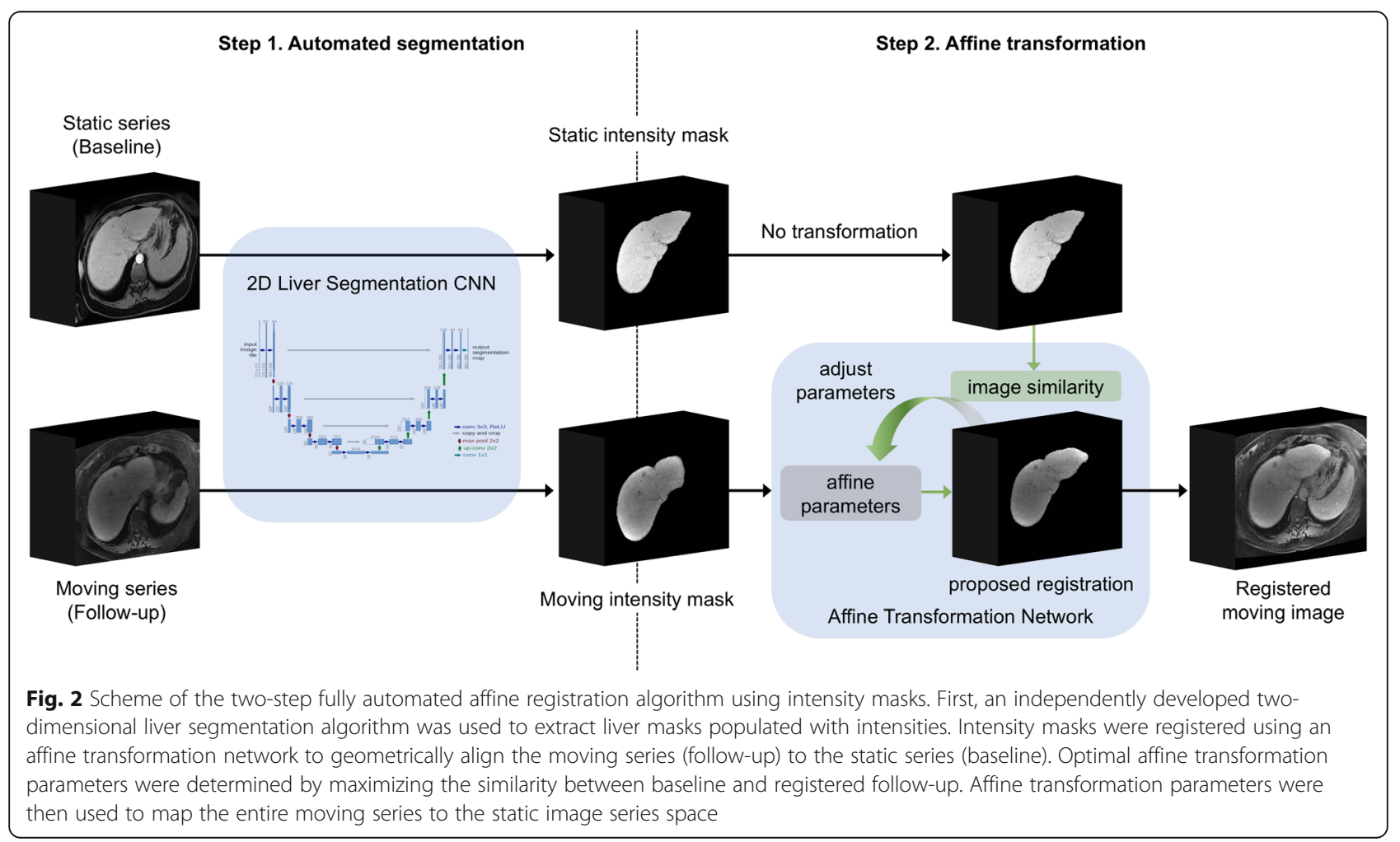


please refer to Additional file 1: S1. The resulting masks were binary: pixels within the mask had values of 1 and pixels outside the mask had values of 0 . Automated registration using the "binary masks" was considered the primary investigational method, since it used native spatial resolution and, lacking any pixel intensity information, has the potential for generalization to multimodality or multiphase registration. A variant of the binary masks called "intensity mask" was then created by multiplying the 3D binary mask by the original raw liver image, thereby populating the masks with the signal intensities from the corresponding image; this was done to investigate how the additional pixel intensity information affected registration performance. Each of the two masks (binary and intensity) was tested as input for the affine transformation network. As a control, the affine transformation was also tested using whole acquired images without any liver segmentation; this was done to determine the effect of focusing the registration on the liver masks on registration accuracy.

\section{Affine transformation network}

The affine transformation was implemented as a neural network with a single 12-neuron dense layer representing 3D affine transformation parameters for translation, rotation, scaling, and shearing. The network estimated affine transformation parameters that optimized alignment between the moving liver mask (i.e., binary or intensity mask) and the static liver mask. Using these transformation parameters, the original, unmasked moving series was transformed to the static series space. A similar process was used for registering whole images (i.e., without segmentation), except all transformations and mappings were done using moving and static whole images rather than moving and static masks. For additional details on the implementation of the affine transformation network, please refer to Additional file 1: S1.

\section{Small-scale reader substudy}

To evaluate how the automated algorithm with binary mask compares to radiologist-performed manual registration and to automated registration with different masks or no masks, we randomly selected 100 inter-exam series pairs from the internal imaging dataset and compared performance using three metrics (Fig. 3).

\section{Manual registration of static and moving series}

Two expert readers (fellowship-trained body radiologists [A.H. and S.I.], each with 5 years of experience in liver imaging), independently, manually registered 100 series pairs (whole images) in the $z$-direction using a commercially available DICOM viewer (Osirix, Geneva, Switzerland) to reflect common clinical practice. Readers registered the images by aligning slices depicting the right portal vein bifurcation in both series as an internal landmark. Slice numbers corresponding to the aligned slices were recorded for each series for subsequent analyses described below.

\section{Manual annotation of focal liver observations on static and moving series}

In the same reading session, the two readers independently annotated any detected focal liver observation on each of the 100 series pairs (static and moving series, separately). Usually, these were distinctive hypointense round nodules. Each annotation included a marker indicating the spatial coordinates of the observation center. After observations were annotated in all image pairs, readers measured the single smallest and largest ones to obtain observations size range.

\section{Fully automated affine registration}

The automated registration algorithm (using mask variations and whole images) was applied to the 100 inter-exam series pairs. Affine transformation parameters from the 100 automated registrations were saved for use in subsequent analyses.

\section{Manual liver segmentation to create ground-truth liver masks}

A senior radiologist (G.M.C.) with 10 years of experience in liver imaging manually segmented the livers on the same 100 series pairs (static and moving series, separately) to create ground-truth liver masks. As described below, these ground-truth liver masks were used to compare the accuracy of automated versus manual registration.

\section{Comparison of manual and automated registration}

Each radiologist's observation annotations were overlain on the ground-truth liver masks, preserving their exact coordinates, to create "annotated masks." To create auto-registered series pairs, the saved affine transformation parameters were applied to the "annotated moving masks." To create manually registered series pairs, the annotated masks were aligned in the $z$-direction using the slice positions selected by each reader as described above. The moving masks were then scaled, rotated, and translated (rigid transformations) to correct for differences in field-of-view or patient positioning from the static masks. This was done to maximize the accuracy of manual registration in the performance comparisons below. Three metrics were used to assess and compare the accuracy of the manual and automated registrations: (1) Liver overlap score (Jaccard index) [30] between the static and registered moving liver masks over the entire liver volume (a score of 1 indicates perfect liver overlap); (2) Image correlation (mutual information) [31] between 
a

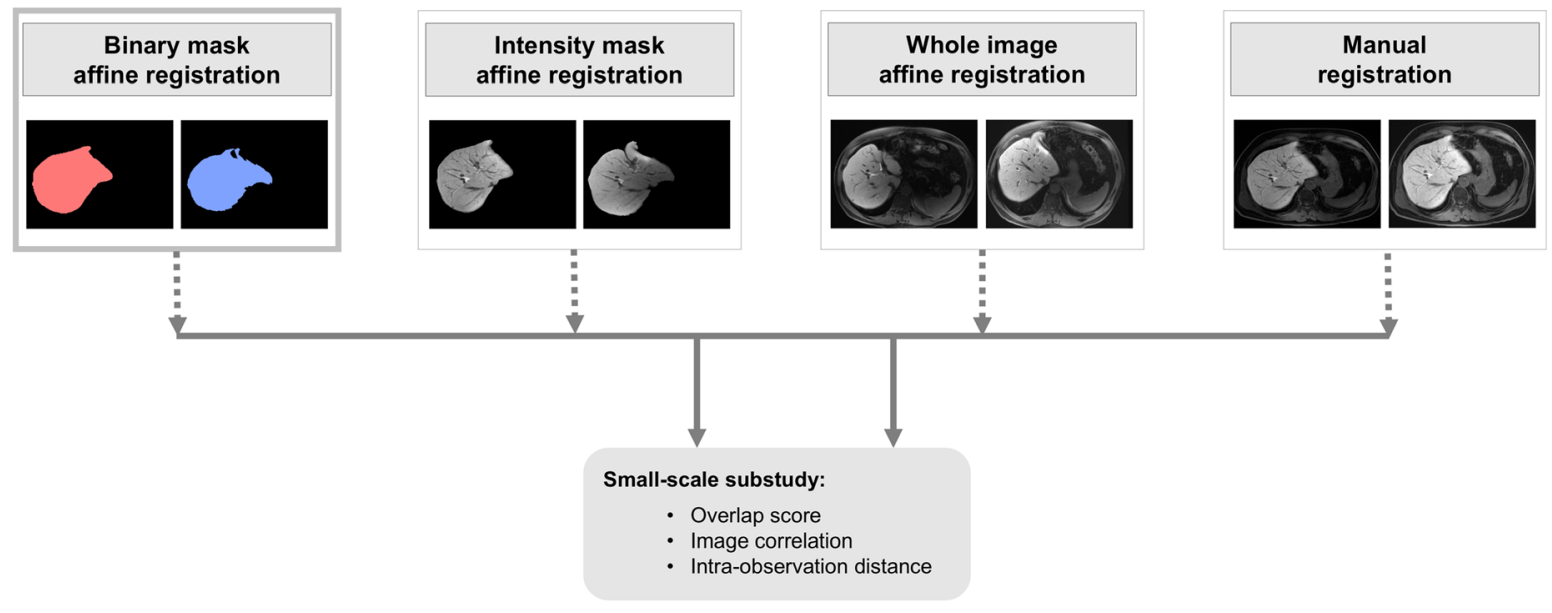

b

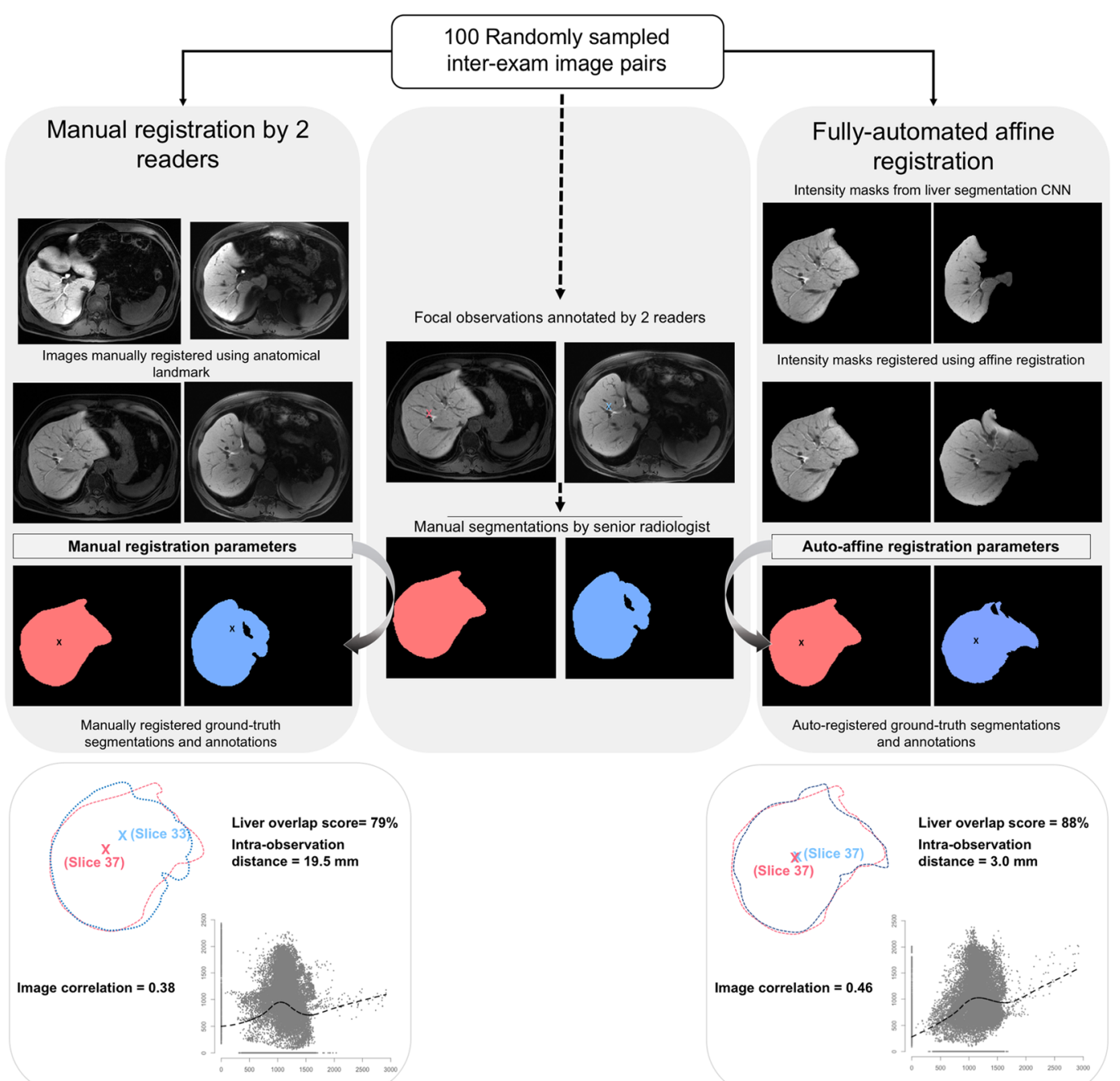

Fig. 3 (See legend on next page.) 
(See figure on previous page.)

Fig. 3 Small-scale substudy (a): Registration methods and performance metrics. Binary mask (first, left to right) was considered the primary investigational method for registration and it was compared to alternate masking method, i.e., whole image registration and manual registration. Small-scale reader substudy (b): flow and performance comparisons between radiologist-performed manual registration and automated algorithm (intensity masks displayed). Three metrics: liver overlap score (Jaccard index), image correlation (mutual information), and intra-observation distance

the ground-truth static intensity mask and the registered ground-truth moving intensity mask (a larger image correlation indicates better registration performance; of note, since image correlation relies on the pixel intensity distribution of the series for calculation, we used image correlation to make only paired comparisons, i.e., we would have been unable to determine if differences in image correlation were attributed to algorithm performance or pixel distributional differences between unpaired groups); and (3) 3D intra-observation distance (marker of focal liver observation co-localization) in millimeter between matched observation centers on overlain static and registered moving series (a score of $0 \mathrm{~mm}$ indicates perfect observation co-localization; in addition to quantitative metrics, a separate analysis qualitatively comparing manual registration and binary mask registration using reader confidence scores for image similarity assessment was performed. Details on this analysis are described in Additional file 1: S2.

\section{Large-scale and external validation substudies}

Since the intensity mask was not meaningfully superior to the binary mask in the small-scale reader study (see results) and since binary masks were considered the primary investigational method, the subsequent studies focused on binary masks. The automated registration algorithm (using binary masks) was applied to all 2,225 within-patient series pairs in the internal dataset to evaluate scalability and to all 438 intra-exam series pairs in the external dataset to evaluate generalizability. Two metrics were used to evaluate registration accuracy: (1) Liver overlap score and (2) image correlation. Intra-observation distance was not assessed as it was not feasible to manually annotate individual observations on the large number $(n=2,663)$ of series in these studies. Similarly, since ground-truth manual segmentation was not feasible for all series pairs, liver overlap score and image correlation were calculated using the masks produced by the CNN liver segmentation algorithm. Computation time was recorded.

\section{Feasibility of cross-modality and multiphase registration}

The registration algorithm (using binary masks) was applied to a single cross-modality series pair (CT to MRI) and a single intra-exam series pair (HBP to arterial phase MRI) as proof-of-concept examples of cross-modality and multiphase registration, respectively. After registration, liver overlap score (Jaccard index) [30] between the static and registered moving liver masks over the entire liver volume was calculated.

\section{Statistical analysis}

All statistical analyses were performed by a biostatistician using R v3.4.0 software [32]. Descriptive summaries were prepared. In the small-scale reader study, the effect of HBP adequacy on liver segmentation accuracy (liver overlap score) was evaluated using linear mixed effects models. Registration metrics achieved by automated registration with the binary mask (the primary investigational method) was compared using paired Bonferroni-corrected $t$ tests to those achieved by every other method (manual registration by each reader, automated registration with intensity mask, and automated registration without any mask [whole image]). Additionally, readers were compared to each other. In the large-scale study, intra-exam and inter-exam differences were compared using unpaired $t$ tests. Influence of patient demographics, inter-series time intervals, imaging artifacts, reader consensus-determined HBP adequacy, and voxel volume differences on one registration metric (liver overlap score) achieved by the primary automated registration algorithm (binary masks) was evaluated in univariate and multivariate analyses, using linear mixed effects models to account for within-patient dependencies. Significant characteristics in the multivariate analysis were determined by backward elimination. Ninety-five percent confidence intervals (CIs) were analytically calculated as appropriate. The $p$ values lower than 0.05 were defined as significant for $t$ tests and linear mixed effects models.

\section{Results}

\section{Small-scale reader substudy}

Performance metrics (mean \pm standard deviation) for the automated and manual registrations in the small-scale study are summarized in Table 2; paired mean differences and their Bonferroni-corrected $p$ values are listed in Additional file 1: Table S1. Figures 4, 5 and 6 provide examples of higher qualitative spatial concordance on auto-registered series over manually registered series.

\section{Liver segmentation accuracy}

Mean liver overlap score between predicted and groundtruth liver masks was $0.96 \pm 0.04$. Liver overlap scores were not significantly different between series with adequate and suboptimal HBP contrast uptake $(p=0.88)$. 
Table 2 Mean \pm standard deviation of performance metrics across 100 within-patient series pair for manual and automated registrations in the small-scale substudy

\begin{tabular}{|c|c|c|c|c|c|}
\hline Registration method & & $\begin{array}{l}\text { Liver overlap } \\
\text { score }\end{array}$ & $\begin{array}{l}\text { Image } \\
\text { correlation }\end{array}$ & $\begin{array}{l}\text { Observation distance (annotated } \\
\text { by reader } 1 \text { ) }\end{array}$ & $\begin{array}{l}\text { Observation distance (annotated } \\
\text { by reader } 2 \text { ) }\end{array}$ \\
\hline \multirow[t]{2}{*}{ Manual registration } & Reader 1 & $0.74 \pm 0.10$ & $0.36 \pm 0.14$ & $14.73 \pm 8.20$ & I \\
\hline & Reader 2 & $0.73 \pm 0.12$ & $0.35 \pm 0.14$ & / & $16.09 \pm 8.49$ \\
\hline \multirow[t]{2}{*}{$\begin{array}{l}\text { Automated registration (with } \\
\text { masking) }\end{array}$} & $\begin{array}{l}\text { Binary } \\
\text { mask }\end{array}$ & $0.86 \pm 0.06$ & $0.42 \pm 0.16$ & $8.40 \pm 4.62$ & $8.42 \pm 5.78$ \\
\hline & $\begin{array}{l}\text { Intensity } \\
\text { mask }\end{array}$ & $0.86 \pm 0.06$ & $0.43 \pm 0.16$ & $8.26 \pm 4.64$ & $8.49 \pm 5.67$ \\
\hline $\begin{array}{l}\text { Automated registration } \\
\text { (without masking) }\end{array}$ & $\begin{array}{l}\text { Whole } \\
\text { image }\end{array}$ & $0.73 \pm 0.15$ & $0.39 \pm 0.17$ & $22.95 \pm 24.42$ & $18.11 \pm 16.06$ \\
\hline
\end{tabular}

Statistical comparisons between registration methods were performed using paired $t$ tests (Additional file 1: Table S1)

\section{Liver overlap score}

On manually registered series, mean liver overlap score was 0.73 or 0.74 , depending on the reader; these differences were not significant. Compared to manual registration, automated registration with binary mask improved the mean liver overlap score from $0.73-0.74$ by $0.11-0.12$ to 0.86 , depending on the reader. In pairwise comparisons, binary mask and intensity mask showed similar overlap scores (mean liver overlap 0.86) and were not statistically different. Automated registration without the initial liver segmentation (i.e., using whole images) was inferior (mean liver overlap 0.73) to binary mask (adjusted $p<0.001$ ) and did not improve mean liver overlap score compared to manual registration.

\section{Image correlation}

On manually registered series, mean image correlation was 0.35 or 0.36 , depending on the reader; these differences were not significant. Compared to manual registration, automated registration with binary mask improved the mean image correlation from $0.35-0.36$ by $0.06-0.07$ to 0.42 , depending on the reader. In pairwise comparisons, binary mask yielded statistically lower image correlation than intensity mask $(0.42$ versus 0.43 , adjusted $p<0.001)$. Automated registration with whole images provided significantly lower image correlation than automated registration using binary masks ( 0.39 versus 0.42 , adjusted $p<0.001)$.

\section{Observation distance}

One reader detected and annotated 139 observations (range 2-68 $\mathrm{mm}$ ), and the other reader 147 observations (range $2-70 \mathrm{~mm}$ ). On manually registered series, mean observation distances were $14.7 \mathrm{~mm}$ and $16.1 \mathrm{~mm}$, depending on the reader; these differences were not significant $(p=0.47)$. Compared to manual registration, automated registration with binary mask decreased the mean intra-observation distance by $6.3-7.7$ to $8.4 \mathrm{~mm}$, depending on the reader. In pairwise comparisons, binary mask was not statistically or meaningfully superior to any other mask. Automated registration without a

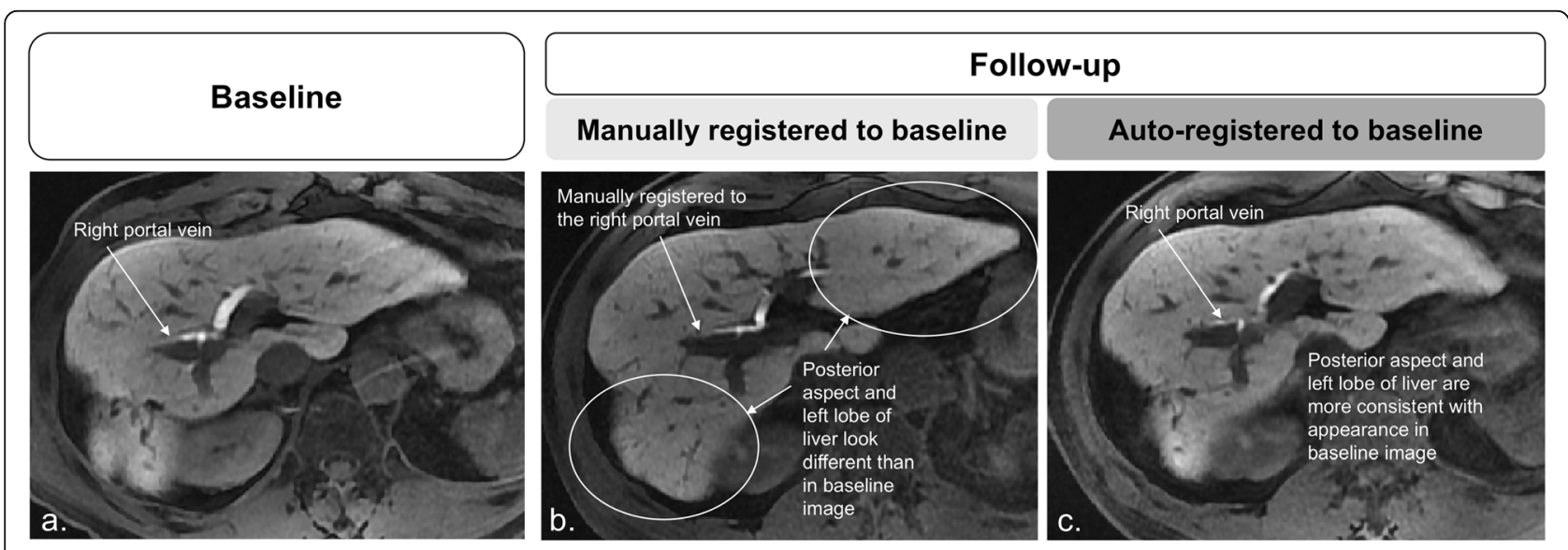

Fig. 4 Baseline static (a) and follow-up moving images $(\mathbf{b}, \mathbf{c})$. In $\mathbf{b}$, the follow-up image registered to baseline manually using the bifurcation of the right portal vein as anatomical reference: differences in liver morphology are pronounced in the posterior aspect of the liver (circle) and in the left lobe (circle). In c, the follow-up image registered to baseline using the automated affine algorithm shows better correspondence to baseline image 


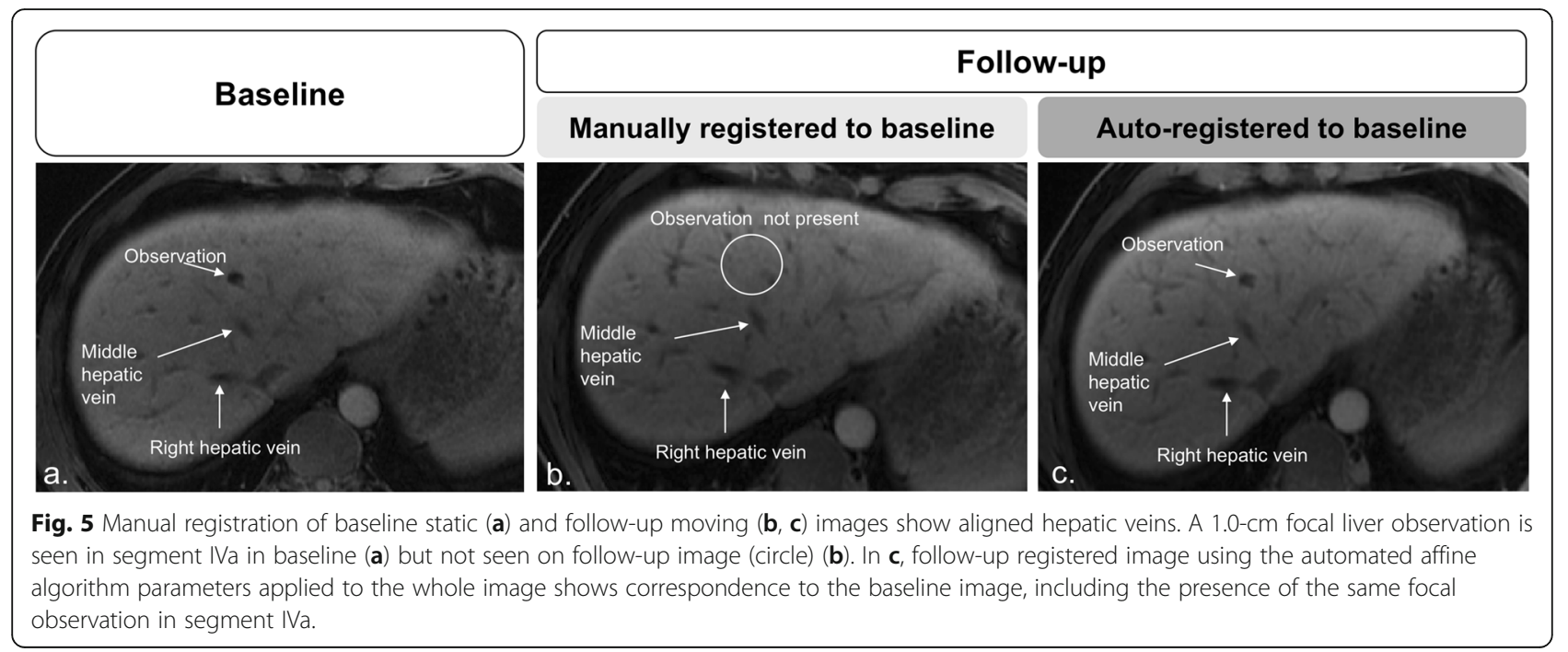

mask (i.e., using whole images) provided mean liver observation distances of 18 and $23 \mathrm{~mm}$; these values were significantly inferior to automated registration with binary mask $(p<0.001)$.

The automated registration algorithm improved qualitative readers' confidence scores for image similarity over standard manual registration as described in Additional file 1: Figure S1.
Large-scale and external validation substudies

Liver overlap and image correlation means \pm standard deviations achieved by automated registration with a binary mask in the large-scale and external validation studies are shown in Table 3. For the large-scale study, mean liver overlap score was 0.81 for inter-exam registration and 0.88 for intra-exam registration while mean image correlation was 0.41 for inter-exam registration and 0.44 for intra-exam

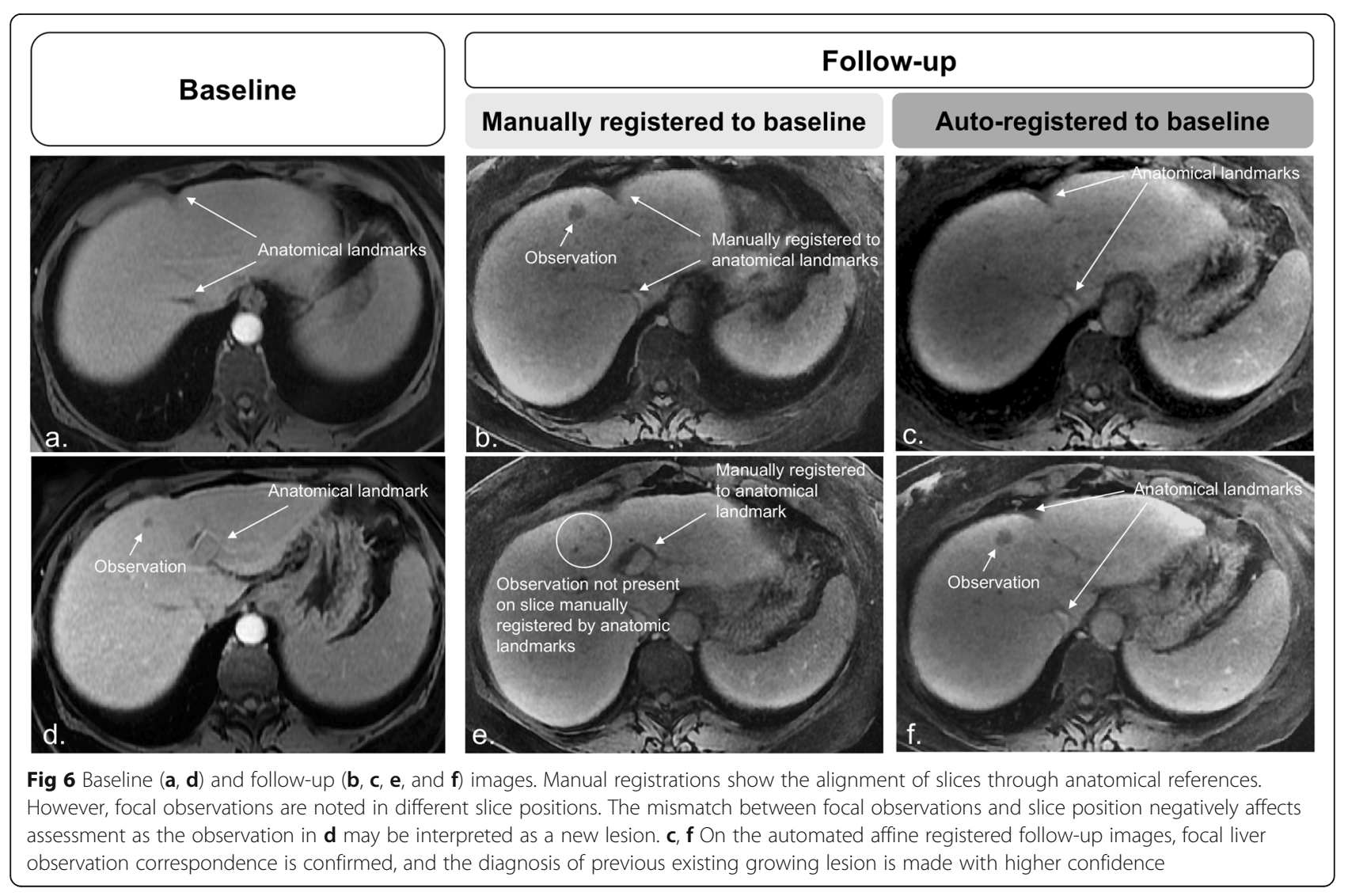


Table 3 Performance analysis (mean \pm standard deviation) of all within-patient pair combinations $(2,225)$ of hepatobiliary phase images in the internal and external datasets (438 image pairs)

\begin{tabular}{lll}
\hline Dataset & Liver overlap score & Image correlation \\
\hline Internal dataset (intra-exam) & $0.88 \pm 0.14$ & $0.44 \pm 0.15$ \\
Internal dataset (inter-exam) & $0.81 \pm 0.14$ & $0.41 \pm 0.14$ \\
External dataset (intra-exam) & $0.91 \pm 0.06$ & $0.41 \pm 0.13$ \\
\hline
\end{tabular}

Statistical comparisons between internal datasets were performed using unpaired $t$ tests

registration; differences between intra-exam and inter-exam registrations were significant $(p<0.001)$. For the external validation study, mean liver overlap score was 0.91 and mean image correlation was 0.41 . Mean computation time for inter-exam binary mask registration (54.6 s) was significantly longer than intra-exam binary mask registration (50.3 s) $(p<0.001)$.

Table 1 summarizes the influence of patient factors, inter-series time intervals, image artifacts, and inter-series voxel volume differences on liver overlap achieved by automated registration with a binary mask in the internal dataset. In multivariate analyses, older age, female sex, greater inter-series time interval, and greater inter-series voxel volume differences independently reduced the liver overlap score. Additionally, liver overlap score was reduced when registering two series with different HBP contrast uptake adequacy scores.
Feasibility of cross-modality and multiphase registration Feasibility of cross-modality and multiphase registration using binary masks is shown in Fig. 7. Cross-modality (CT to MRI) liver overlap score was 0.93 and multiphase (arterial phase to hepatobiliary phase MRI) liver overlap score was 0.89 using the proposed automated method.

\section{Discussion}

In this study, we applied a novel fully automated affinebased registration algorithm to MRI T1-weighted HBP series acquired at different delays from contrast injection within the same exam and at different exam dates and compared its performance against standard manual registration. The automated algorithm produced better registration of the liver between series over manual registration and, most importantly, better co-localization of focal observations.

In clinical practice, image registration is commonly performed manually, which confines the registration to magnification and to alignment in the $z$-direction. This means that the radiologist may be able to scroll up or down, zoom in or out, to find the slice that most closely aligns to the comparison image, but cannot alter the image in any other plane. Rotational alignments can be performed using multiplanar reformation software addons, often time-consuming and difficult to perform. Our proposed $3 \mathrm{D}$ automated registration saves time and

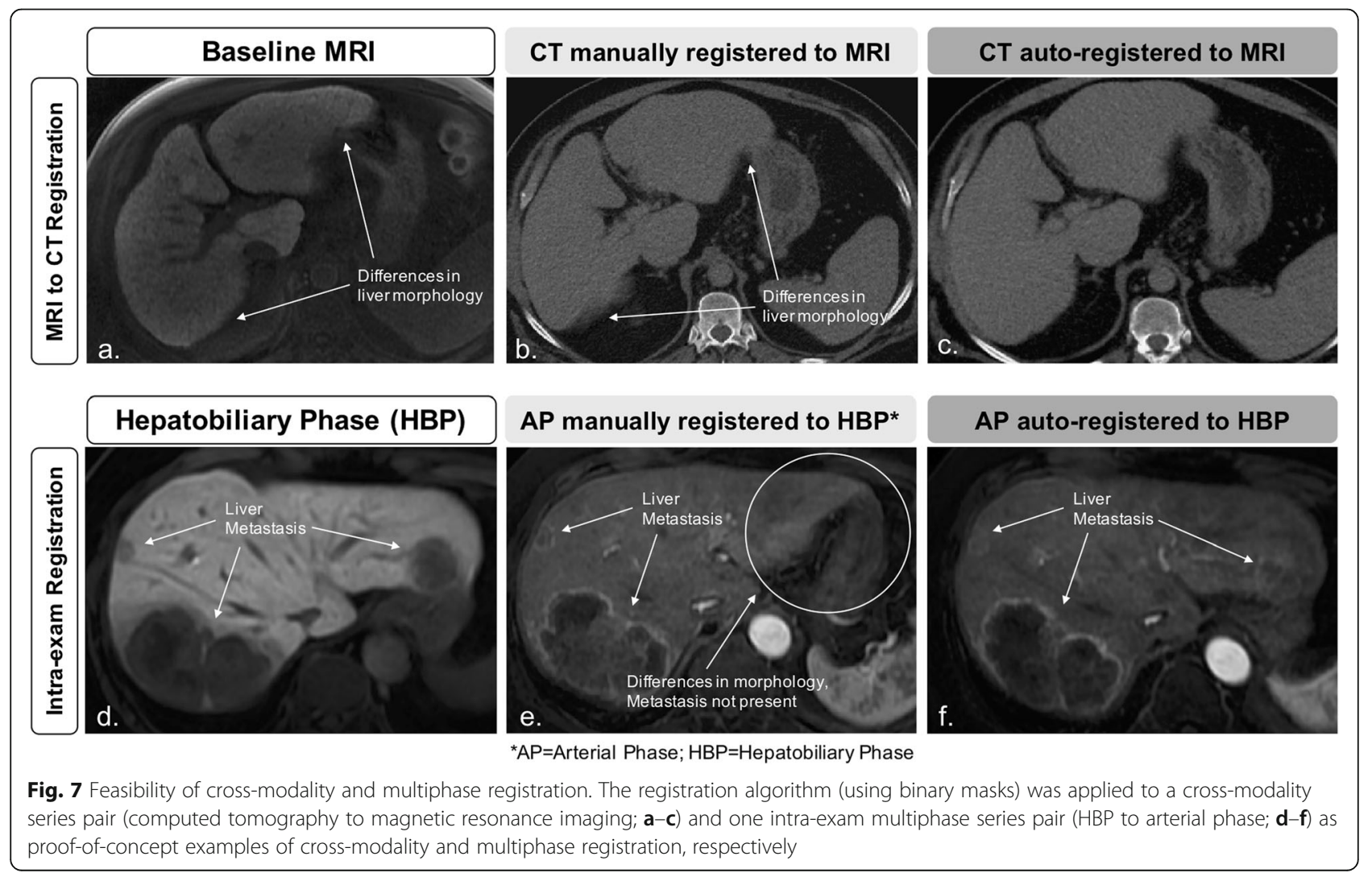


mitigates cross-sectional misalignment attributed to changes in position or dynamic liver morphology between series, as the CNN-based liver segmentations/ masks focus the affine transformations to the organ of interest, which, as shown by others and verified in this study, improves accuracy [14].

Compared to masks that retained intensity information, binary masks achieved overall similar performance, except for small and likely unmeaningful differences in image correlation, suggesting that the liver masking (not the liver intensities) drives registration accuracy. Since the affine transformation does not meaningfully benefit from intensity information, the proposed algorithm using binary masks does not rely on a particular imaging sequence, phase, or modality and can potentially be extended to cross-modality and multiphase registration tasks. Finally, we showed the proposed algorithm is scalable and generalizable across a large number of exams and different populations and scanners, achieving high liver overlap scores and image correlations across over 2,500 series pairs.

The performance of the proposed automated registration algorithm was not affected by patient body mass index, liver disease etiology, and imaging artifacts. Interexam registration performance was significantly affected by the time between series; compared to intra-exam series, average liver overlap was reduced by $10 \%$ when images were acquired more than 2 years apart. This was expected since the liver may change in volume and shape over prolonged time periods, especially in patients with chronic liver disease [33, 34]. Differences in voxel volume between series also reduced registration performance, probably because such differences force the $3 \mathrm{D}$ registration to interpolate the moving series across a different anisotropic space than the static image. Differences in HBP contrast uptake adequacy within a series pair reduced registration performance, but the reduction was small and likely not clinically relevant. We believe this minor decrease in registration performance could be related to small differences in liver mask predictions between adequate and suboptimal series, where small isointense vessels in suboptimal series may be included in the liver masks. Registration performance was not affected when both series in a pair were either adequate or suboptimal. Older age and female sex each mildly decreased registration accuracy. We speculate that different breath-hold capabilities or breathing patterns across age and sex [35] could affect variability in liver position and shape between acquisitions. Further research is needed to confirm and elucidate the mechanism for these findings.

The incorporation of segmentation techniques to constrain registration focus on the organ of interest and improve performance over whole image registration has been proposed $[4,9,14,18,19]$. However, these often relied on laborious manual or semiautomated segmentation and/ or were validated on small cohorts [9, 11, 14, 17]. Being completely automated, and hence, less time-intensive than semiautomated or manual methods, our registration algorithm can be applied to a large set of image series pairs while achieving greater accuracy than whole image registration (i.e., without segmentation). Additionally, most prior works proposed deformable techniques to address the nonlinear morphology of the liver $[9,10,17,18]$. Although achieving good performance overall, deformable registration can be unreliable and inadvisable for diagnostic purposes since distortions in organ or lesion appearance may lead to erroneous measurements or characterization $[13,25,26]$. Hence, some investigators favor non-deformable image registration for lesion evaluation and follow-up $[25,26]$. Carrillo et al. [11] and Fujioka et al. [19] proposed rigid registration of liver images to evaluate locoregional therapy response with promising results, reporting mean registration errors ranging from 3.05 to $13.00 \mathrm{~mm}$. Although similar to the intra-observation distances reported in our study, results were reported on small cohorts ( $<20$ patients) and relied on extensive image preprocessing, manual registration, and/or manual liver segmentations, possibly limiting application to busy clinical settings [11, 19]. Additionally, Carrillo et al. [11] reported the exclusion of slices close to the diaphragm in the reference volumes as well as the exclusion of 2 out of 17 individuals due to low-image quality (low signal-to-noise ratio [SNR] or motion artifacts). Overall, our algorithm was not affected by the presence of imaging artifacts, likely due to the robustness of our liver segmentation algorithm.

Foruzan and Motlagh [14] have used a multistep approach to register liver images for interventional therapy purposes. In their study, a semiautomated liver segmentation was followed by a rigid and a non-rigid registration. Their liver segmentation achieved slight lower overlap (0.93) than our CNN-based liver segmentation. For their rigid and non-rigid registration algorithms, mean liver overlap and intra-observation distance were 0.75 and 11.7 $\mathrm{mm}$ and 0.78 and $10.11 \mathrm{~mm}$, respectively. These values are slightly worse than those achieved by our algorithm. Although the robustness of our $\mathrm{CNN}$-based liver segmentation may in part be the reason why we found slightly better results, their study performed multimodality registration on low-field strength MR data with very low SNR images, making their registration task more challenging.

Conversely, Fernandez-de-Manuel et al. [36] proposed a liver-focused deformable registration algorithm using high SNR gadoxetate disodium-enhanced 3D T1-weighted HBP images for liver lesion evaluation. In their study, they reported a mean intra-observation distance of $7.07 \mathrm{~mm}$ after registration, which is similar to our results. However, since they used a deformable registration technique, a direct comparison to our results may warrant some caution. 
Unfortunately, we could not find any publicly available datasets or CNN-segmentation-based algorithms for liver registration to test our approach and/or perform a head-to-head comparison. Our proposed algorithm also achieved shorter computation times than previously proposed liver registration algorithms, which ranged from under $2 \mathrm{~min}$ [14] to 2 to $30 \mathrm{~min}[8,9]$. However, faster computation times may be attributed to technological advances in computing (using graphics processing units) and differences in the registration task (affine versus deformable). Hence, it is likely the computation time reported in our study will be easily overcome as technology advances in the next few years.

A limitation of our study was its retrospective design, which precluded the assessment of how our algorithm would impact radiologist performance in a clinical setting. Additionally, our cohort was comprised mostly of patients with chronic liver disease and under surveillance for HCC. Hence, the number of patients with advanced neoplastic disease, locoregional treatment, or liver resections was small. Therefore, we could not assess how major alterations in liver size and shape related to these procedures would impact the registration. Finally, since ground-truth manual segmentations were not feasible for all 2,663 series pairs used in the large-scale and external validation studies, liver overlap score and image correlation were calculated using the masks produced by the $\mathrm{CNN}$ liver segmentation algorithm.

In conclusion, our proposed two-step fully automated affine registration algorithm accurately registers the liver within and between examinations and yields significantly better overall liver and focal observation co-localization compared to the manual alignment commonly performed in clinical practice.

\section{Additional files}

Additional file 1: S1. Fully-automated affine registration algorithm. S2. Reader confidence study for qualitative image similarity assessment. Table S1. Paired comparisons for the small-scale reader substudy. Figure S1. Reader confidence scores for qualitative image similarity assessment. (DOCX 10388 kb)

\section{Abbreviations}

3D: Three-dimensional; CNN: Convolutional neural network; CT: Computed tomography; HBP: Hepatobiliary phase; MRI: Magnetic resonance imaging

\section{Acknowledgements \\ The authors acknowledge Diagnósticos da América SA (DASA) for the research collaboration in providing the external image dataset.}

\section{Authors' contributions}

$\mathrm{KH}$ and GMC provided a substantial and equal contribution to the conception and design of the study, interpretation of the results, and drafting and reviewing the manuscript. These authors should be listed as cofirst authors since this work could only have been possible with the complementary and synergistic contributions of these authors. $\mathrm{KH}$ was also responsible for algorithm development. GMC also provided the clinical expertise necessary for the completion of this work. $\mathrm{AH}$ and $\mathrm{SI}$ contributed as expert readers for image analysis and in the revision of the manuscript. KW, TD, RB, and AS have made a substantial contribution to the data collection and analysis, as well as an extensive revision of the final manuscript. LKB contributed to this work by collecting all the data regarding the external validation analysis and has reviewed the final version of this manuscript. AS, KJF, AH, and CBS provided guidance as senior authors, made a significant contribution to the design of the study, and equally and substantially revised the final manuscript. All authors read and approved the final manuscript.

\section{Funding}

The authors state that this work did not receive any funding.

\section{Availability of data and materials}

The datasets used and/or analyzed during the current study are available from the corresponding author on reasonable request

\section{Ethics approval and consent to participate}

The study was HIPAA-compliant and approved by the institutional review boards with waived informed consent requirement (UC San Diego Human Research Protections Program (\#071983, \#171538)

\section{Consent for publication}

Not applicable

\section{Competing interests}

The authors declare that they have no competing interests.

\section{Author details}

${ }^{1}$ Liver Imaging Group, Department of Radiology, University of California San Diego, La Jolla, CA, USA. AiDA Laboratory, Department of Radiology, University of California San Diego, La Jolla, CA, USA. ${ }^{3}$ Department of Radiology, Stanford University, Palo Alto, CA, USA. ${ }^{4}$ Abdominal and Pelvic MRI, Radiology, CDPI Clinics, DASA Company, Fluminense Federal University (UFF), Rio de Janeiro, Brazil. ${ }^{5}$ Department of Family Medicine and Public Health, University of California San Diego, La Jolla, CA, USA. ${ }^{6}$ Altman Clinical Translational Research Institute, 9452 Medical Center Drive, Lower Level 501, La Jolla, CA 92037, USA.

Received: 24 May 2019 Accepted: 28 August 2019

Published online: 26 October 2019

\section{References}

1. https://www.acr.org/Clinical-Resources/Reporting-and-Data-Systems/LIRADS/CT-MRI-LI-RADS-V2018. Accessed 24 Jan 2019.

2. Blackall JM, King AP, Penney GP, Adam A, Hawkes DJ (2001) A statistical model of respiratory motion and deformation of the liver. In: Niessen W, Viergever M (Eds.): MICCAI 2001, LNCS 2208. Springer, Berlin, Heidelberg, pp 1338-1340

3. Blackall JM, Ahmad S, Miquel ME, McClelland JR, Landau DB, Hawkes DJ (2006) MRI-based measurements of respiratory motion variability and assessment of imaging strategies for radiotherapy planning. Phys Med Biol 51:4147-4169. https://doi.org/10.1088/0031-9155/51/17/003

4. Vivanti R, Szeskin A, Lev-Cohain N, Sosna J, Joskowicz L (2017) Automatic detection of new tumors and tumor burden evaluation in longitudinal liver $C T$ scan studies. Int J Comput Assist Radiol Surg 12:1945-1957. https://doi.org/10.1007/s11548-01 7-1660-z

5. Shimizu S, Shirato H, Xo B et al (1999) Three-dimensional movement of a liver tumor detected by high-speed magnetic resonance imaging. Radiother Oncol 50:367-370. https://doi.org/10.1016/S0167-8140(98)00140-6

6. von Siebenthal M, Szekely G, Gamper U, Boesiger P, Lomax A, Cattin P (2007). 4D MR imaging of respiratory organ motion and its variability. Phys Med Biol 52:1547-1564 doi:https://doi.org/10.1088/0031-9155/52/6/001

7. Zhang Y, Boye D, Tanner C, Lomax AJ, Knopf A (2012) Respiratory liver motion estimation and its effect on scanned proton beam therapy. Phys Med Biol 57:1779-1795. https://doi.org/10.1088/0031-9155/57/7/1779

8. Wilson DL, Carrillo A, Zheng L, Genc A, Duerk JL, Lewin JS (1998) Evaluation of 3D image registration as applied to MR-guided thermal treatment of liver cancer. J Magn Reson Imaging 8:77-84. https://doi.org/10.1002/jmri.1880080117

9. Brock KK, Dawson LA, Sharpe MB, Moseley DJ, Jaffray DA (2006) Feasibility of a novel deformable image registration technique to facilitate classification, targeting, and monitoring of tumor and normal tissue. Int J Radiat Oncol Biol Phys 64:12451254. https://doi.org/10.1016/j.jirobp.2005.10.027 
10. Rohlfing T, Maurer CR Jr, O'Dell WG, Zhong J (2004) Modeling liver motion and deformation during the respiratory cycle using intensity-based nonrigid registration of gated MR images. Med Phys 31:427-432. https:/doi.org/10.1118/1.1644513

11. Carrillo A, Duerk JL, Lewin JS, Wilson DL (2000) Semiautomatic 3-D image registration as applied to interventional MRI liver cancer treatment. IEEE Trans Med Imaging 19:175-185. https://doi.org/10.1109/42.845176

12. Erickson BJ, Patriarche J, Wood C et al (2007) Image registration improves confidence and accuracy of image interpretation. Cancer Inform 4:19-24

13. Zitová B, Flusser J (2003) Image registration methods: a survey. Image Vis Comput 21:977-1000. https://doi.org/10.1016/S0262-8856(03)00137-9

14. Foruzan AH, Motlagh HR (2015) Multimodality liver registration of Open-MR and CT scans. Int J Comput Assist Radiol Surg 10:1253-1267. https:/doi.org/10.1007/ s11548-014-1139-0

15. Gunay G, Luu MH, Moelker A, van Walsum T, Klein S (2017) Semiautomated registration of pre-and intraoperative CT for imageguided percutaneous liver tumor ablation interventions. Med Phys 44: 3718-3725. https://doi.org/10.1002/mp.12332

16. Luu HM, Klink C, Niessen W, Moelker A, Walsum Tv (2016). Non-rigid registration of liver $\mathrm{CT}$ images for $\mathrm{CT}$-guided ablation of liver tumors. Plos One, 11(9):e0161600. https://doi.org/10.1371/journal.pone.0161600

17. Xie Y, Chao M, Xiong G (2011). Deformable image registration of liver with consideration of lung sliding motion. Med Phys, 38(10):5351-5361. doi: org/ https://doi.org/10.1118/1.3633902

18. Voroney JP, Brock KK, Eccles C, Haider M, Dawson LA (2006) Prospective comparison of computed tomography and magnetic resonance imaging for liver cancer delineation using deformable image registration. Int J Radiat Oncol Biol Phys 66:780-791. https://doi.org/10.1016/j.jirobp.2006.05.035

19. Fujioka C, Horiguchi J, Ishifuro $M$ et al (2006) A feasibility study: evaluation of radiofrequency ablation therapy to hepatocellular carcinoma using image registration of preoperative and postoperative CT. Acad Radiol 13:986-994. https://doi.org/10.1016/j.acra.2006.05.011

20. Lu F, Wu F, Hu P, Peng Z, Kong D (2017) Automatic 3D liver location and segmentation via convolutional neural network and graph cut. Int J Comput Assist Radiol Surg 12:171-182. https://doi.org/10.1007/s11548016-1467-3

21. Hu P, Wu F, Peng J, Bao Y, Chen F, Kong D (2017) Automatic abdominal multi-organ segmentation using deep convolutional neural network and time-implicit level sets. Int J Comput Assist Radiol Surg 12:399-411. https:// doi.org/10.1007/s11548-016-1501-5

22. Hu Y, Modat M, Gibson E et al (2018) Weakly-supervised convolutional neural networks for multimodal image registration. Med Image Anal 49:113. https://doi.org/10.1016/j.media.2018.07.002

23. de Vos BD, Berendsen FF, Viergever MA, Staring M, Išgum I (2017) End-toend unsupervised deformable image registration with a convolutional neural network. In: Cardoso M et al (eds) Deep Learning in Medical Image Analysis and Multimodal Learning for Clinical Decision Support. Lecture Notes in Computer Science, vol 10553. Springer, Cham, pp 204-212

24. Eppenhof KA, Lafarge MW, Moeskops P et al (2018) Deformable image registration using convolutional neural networks. In: Medical Imaging 2018 : Image Processing, vol 10574, p. 105740S. International Society for Optics and Photonics. https://doi.org/10.1117/12.2292443

25. Fortin D, Basran PS, Berrang T, Peterson D, Wai ES (2014). Deformable versus rigid registration of $\mathrm{PET} / \mathrm{CT}$ images for radiation treatment planning of head and neck and lung cancer patients: a retrospective dosimetric comparison. Radiat Oncol 10;9:50. doi: https://doi.org/10.1186/1748-717X-9-50

26. Batchelar D, Collins A, Jaswal J, Schmid M, Bachand G, Crook J (2015) A comparison of rigid to deformable registration for dose escalation to the dominant lesion in HDR prostate brachytherapy. Brachytherapy 14:S50. https://doi.org/10.1016/j.brachy.2015.02.279

27. Cunha GM, Hasenstab KA, Wang K et al (2018) Automated assessment of liver parenchymal Enhancement on hepatobiliary phase MR images using a convolutional neural network. Presented as digital poster at the ISMRM 27th Annual Meeting \& Exhibition in Montreal, Canada, 11-16 May 2019

28. Wang K, Mamidipalli A, Retson T et al (2019). Automated CT and MRI liver segmentation and biometry using a generalized convolutional neural network Radiology 1(2):e180022. doi: https://doi.org/10.1148/ryai.2019180022

29. Github, https://github.com/fchollet/keras. Accessed 7 Jan 2018.

30. Crum WR, Camara O, Hill DL (2006) Generalized overlap measures for evaluation and validation in medical image analysis. IEEE Trans Med Imaging 25:1451-1461. https://doi.org/10.1109/TMI.2006.880587
31. Maes F, Vandermeulen D, Suetens $P$ (2003) Medical image registration using mutual information. Proc IEEE 91:1699-1722. https://doi.org/10.1109/ JPROC.2003.817864

32. R Core Team (2017) R: a language and environment for statistical computing. R Foundation for Statistical Computing, Vienna, Austria. http:// www.R-project.org/

33. Schmucker DL (2005). Age-related changes in liver structure and function: implications for disease? Exp Gerontol 40(8-9), 650-659. https://doi.org/10.1 016/j.exger.2005.06.009

34. Ito K, Mitchell DG (2000) Hepatic morphologic changes in cirrhosis: MR imaging findings. Abdom Imaging 25:456-461. https://doi.org/10.1007/ s002610000013

35. Ragnarsdottir M, Kristinsdottir EK (2006) Breathing movements and breathing patterns among healthy men and women 20-69 years of age. Respiration 73:48-54. https://doi.org/10.1159/000087456

36. Fernandez-de-Manuel L, Wollny G, Kybic J et al (2014) Organ-focused mutual information for nonrigid multimodal registration of liver $C T$ and $\mathrm{Gd}$ EOB-DTPA-enhanced MRI. Med Image Anal 18:22-35. https://doi.org/10.1 016/j.media.2013.09.002

\section{Publisher's Note}

Springer Nature remains neutral with regard to jurisdictional claims in published maps and institutional affiliations.

\section{Submit your manuscript to a SpringerOpen ${ }^{\circ}$ journal and benefit from:}

- Convenient online submission

- Rigorous peer review

- Open access: articles freely available online

High visibility within the field

- Retaining the copyright to your article

Submit your next manuscript at $>$ springeropen.com 